\title{
Approximation of general Pexider functional inequalities in fuzzy Banach spaces
}

\author{
Gang Lu ${ }^{\mathrm{a}}$, Jincheng Xin ${ }^{\mathrm{a}}$, Yuanfeng Jin ${ }^{\mathrm{b}, *}$, Choonkil Park ${ }^{\mathrm{c}}$
}

${ }^{a}$ Department of Mathematics, School of Science, ShenYang University of Technology, Shenyang 110870, P. R. China.

${ }^{b}$ Department of Mathematics, Yanbian University, Yanji 133001, People's Republic of China.

${ }^{c}$ Department of Mathematics, Research Institute for Natural Sciences, Hanyang University, Seoul 04763, Republic of Korea.

\begin{abstract}
In this paper, we investigate a fuzzy version of a generalized Hyers-Ulam-Rassias type stability for the following Pexider functional inequalities

$$
\begin{aligned}
& f(x+y)+f(x-y)+g(z)+h(l) \leqslant k p\left(\frac{2 x+z+l}{k}\right), \\
& f(x+y)+f(x-y)+g(z)+k h(l) \leqslant k p\left(\frac{x+z}{k}+l\right),
\end{aligned}
$$

where $k$ are nonzero real scalars. In the fuzzy normed linear space setting is presented. In this condition, we give an alternative proof of this result in fuzzy Banach space.
\end{abstract}

Keywords: Fuzzy approximation, Pexider functional inequality, fuzzy Banach space.

2010 MSC: 39B62, 39B52, 46B25.

(C)2019 All rights reserved.

\section{Introduction and preliminaries}

The class of approximate solutions of the given functional equation one can ask whether each mapping from this class can be somehow approximated by an exact solution of the considered equation. Such a problem was formulated by Ulam in 1940 ([33]).

In 1941, Hyers [16] gave the first affirmative answer to the question of Ulam for Banach spaces. Let $f: E \rightarrow E^{\prime}$ be a mapping between Banach spaces such that

$$
\|f(x+y)-f(x)-f(y)\| \leqslant \delta
$$

\footnotetext{
*Corresponding author

Email addresses: lvgang1234@hanmail .net (Gang Lu), 1146196932@qq.com (Jincheng Xin), yfkim@ybu.edu.cn (Yuanfeng Jin), baak@hanyang.ac.kr (Choonkil Park)

doi: $10.22436 /$ jnsa.012.04.02
}

Received: 2018-04-02 Revised: 2018-08-05 Accepted: 2018-10-26 
for all $x, y \in E$, and for some $\delta>0$. Then there exists a unique additive mapping $T: E \rightarrow E^{\prime}$ such that

$$
\|f(x)-T(x)\| \leqslant \delta
$$

for all $x \in E$. Moreover, if $f(t x)$ is continuous in $t \in \mathbb{R}$ for each fixed $x \in E$, then $T$ is $\mathbb{R}$-linear. In 1978, Rassias [29] proved the following theorem.

Theorem 1.1. Let $\mathrm{f}: \mathrm{E} \rightarrow \mathrm{E}^{\prime}$ be a mapping from a normed vector space $\mathrm{E}$ into a Banach space $\mathrm{E}^{\prime}$ subject to the inequality

$$
\|f(x+y)-f(x)-f(y)\| \leqslant \epsilon\left(\|x\|^{p}+\|y\|^{p}\right)
$$

for all $x, y \in E$, where $\epsilon$ and $p$ are constants with $\epsilon>0$ and $p<1$. Then there exists a unique additive mapping $\mathrm{T}: \mathrm{E} \rightarrow \mathrm{E}^{\prime}$ such that

$$
\|f(x)-T(x)\| \leqslant \frac{2 \epsilon}{2-2^{p}}\|x\|^{p}
$$

for all $\mathrm{x} \in \mathrm{E}$. If $\mathrm{p}<0$ then inequality (1.1) holds for all $\mathrm{x}, \mathrm{y} \neq 0$, and (1.2) for $\mathrm{x} \neq 0$. Also, if the function $\mathrm{t} \mapsto \mathrm{f}(\mathrm{t} \mathrm{x})$ from $\mathbb{R}$ into $\mathrm{E}^{\prime}$ is continuous in $\mathrm{t} \in \mathbb{R}$ for each fixed $\mathrm{x} \in \mathrm{E}$, then $\mathrm{T}$ is $\mathbb{R}$-linear.

The result of Rassias has influenced the development of what is now called the Hyers-Ulam-Rassias stability theory for functional equations. On the other hand, Rassias [30] generalized the Hyers-Ulam stability result by presenting a weaker condition controlled by a product of different powers of norms.

Theorem 1.2 ([30]). If it is assumed that there exist constants $\Theta \geqslant 0$ and $p_{1}, p_{2} \in \mathbb{R}$ such that $p=p_{1}+p_{2} \neq 1$, and $\mathrm{f}: \mathrm{E} \rightarrow \mathrm{E}^{\prime}$ is a mapping from a norm space $\mathrm{E}$ into a Banach space $\mathrm{E}^{\prime}$ such that the inequality

$$
\|f(x+y)-f(x)-f(y)\| \leqslant \Theta\|x\|^{p_{1}}\|y\|^{p_{2}}
$$

for all $x, y \in E$, then there exists a unique additive mapping $T: E \rightarrow E^{\prime}$ such that

$$
\|f(x)-T(x)\| \leqslant \frac{\Theta}{2-2^{p}}\|x\|^{p}
$$

for all $\mathrm{x} \in \mathrm{E}$. If, in addition, $\mathrm{f}(\mathrm{t} \mathrm{x})$ is continuous in $\mathrm{t} \in \mathbb{R}$ for each fixed $\mathrm{x} \in \mathrm{E}$, then $\mathrm{T}$ is $\mathbb{R}$-linear.

In 1991, Gajda [14] answered the question for the case $p>1$, which was raised by Rassias. In 1994, a generalization of Rassias theorem was obtained by Gǎvruta [15] by replacing the bound $\epsilon\left(\|x\|^{p}+\|y\|^{p}\right)$ by a general control function $\varphi(x, y)$. More generalizations and applications of the Hyers-Ulam stability to a number of functional equations and mappings can be found in $[1,4,6,8-11,17,22,24-26,28,31,32]$. These stability results can be applied in stochastic analysis [23], financial and actuarial mathematics, as well as in psychology and sociology.

In [27], Park et al. investigated the following inequalities

$$
\begin{aligned}
\|f(x)+f(y)+f(z)\| & \leqslant\left\|2 f\left(\frac{x+y+z}{2}\right)\right\|, \\
\|f(x)+f(y)+f(z)\| & \leqslant\|f(x+y+z)\|, \\
\|f(x)+f(y)+2 f(z)\| & \leqslant\left\|2 f\left(\frac{x+y}{2}+z\right)\right\|,
\end{aligned}
$$

in Banach spaces. Recently, several stability results have been recently obtained for various equations, also for mappings with more general domains and ranges see $([7,12,20,21])$. In the paper, we consider the following Pexider functional inequalities

$$
f(x+y)+f(x-y)+g(z)+h(l) \leqslant k p\left(\frac{2 x+z+l}{k}\right),
$$




$$
f(x+y)+f(x-y)+g(z)+k h(l) \leqslant k p\left(\frac{x+z}{k}+l\right),
$$

where $k$ are nonzero real scalars.

In 1984, Katsaras [18] defined a fuzzy norm on a linear space to construct a fuzzy vector topological structure on the space. Later, some mathematicians have defined fuzzy norms on a linear space from different points of view $([13,19,34])$. In 2003, Bag and Samanta [3] modified the definition of Cheng and Mordeson [5] by removing a regular condition. They also established a decomposition theorem of a fuzzy norm into a family of crisp norms and investigated some properties of fuzzy norms (see [2]). Following [2], we give the employing notion of a fuzzy norm.

Let $X$ be a real linear space. A function $N: X \times \mathbb{R} \rightarrow[0,1]$ (the so-called fuzzy subset) is said to be a fuzzy norm on $X$ if for all $x, y \in X$ and all $a, b \in \mathbb{R}$ :

$\left(\mathrm{N}_{1}\right) \mathrm{N}(\mathrm{x}, \mathrm{a})=0$ for $\mathrm{a} \leqslant 0$;

$\left(\mathrm{N}_{2}\right) x=0$ if and only if $\mathrm{N}(\mathrm{x}, \mathrm{a})=1$ for all $\mathrm{a}>0$;

$\left(\mathrm{N}_{3}\right) \mathrm{N}(\mathrm{ax}, \mathrm{b})=\mathrm{N}\left(\mathrm{x}, \frac{\mathrm{b}}{|\mathrm{a}|}\right)$ if $\mathrm{a} \neq 0$;

$\left(N_{4}\right) N(x+y, a+b) \geqslant \min \{N(x, a), N(y, b)\} ;$

$\left(N_{5}\right) N(x,$.$) is a non-decreasing function on \mathbb{R}$ and $\lim _{a \rightarrow \infty} N(x, a)=1$;

$\left(\mathrm{N}_{6}\right)$ for $x \neq 0, N(x,$.$) is (upper semi) continuous on \mathbb{R}$.

The pair $(X, N)$ is called a fuzzy normed linear space. One may regard $N(x, a)$ as the truth value of the statement the norm of $x$ is less than or equal to the real number $a^{\prime}$.

Example 1.3. Let $(X,\|\cdot\|)$ be a normed linear space. Let

$$
N(x, a)= \begin{cases}0, & a \leqslant 0, \\ \frac{a}{\|x\|}, & 0<t \leqslant\|x\|, \\ 1, & t>\|x\| .\end{cases}
$$

Then $N(x, t)$ is a fuzzy norm on $X$ and $(X, N(x, t))$ is a fuzzy normed space.

Definition 1.4. Let $(X, N)$ be a fuzzy normed linear space. Let $x_{n}$ be a sequence in $X$. Then $x_{n}$ is said to be convergent if there exists $x \in X$ such that $\lim _{n \rightarrow \infty} N\left(x_{n}-x, a\right)=1$ for all $a>0$. In that case, $x$ is called the limit of the sequence $x_{n}$ and we denote it by $N-\lim _{n \rightarrow \infty} x_{n}=x$.

Definition 1.5. A sequence $x_{n}$ in $X$ is called Cauchy, if for each $\epsilon>0$ and each $a>0$ there exists $n_{0}$ such that for all $n \geqslant n_{0}$ and all $p>0$, we have $N\left(x_{n+p}-x_{n}, a\right)>1-\epsilon$.

It is known that every convergent sequence in fuzzy normed space is Cauchy. If each Cauchy sequence is convergent, then the fuzzy norm is said to be complete and the fuzzy normed space is called a fuzzy Banach space.

Throughout this paper, assume that $(\mathrm{X}, \mathrm{N})$ is an fuzzy normed space and $(\mathrm{Y}, \mathrm{N})$ is an fuzzy Banach space.

\section{Hyers-Ulam stability of the functional inequality (1.3)}

Throughout this section, assume that $a, b, c$ and $k$ are the nonzero scalars.

Proposition 2.1. Let $\mathrm{f}, \mathrm{g}, \mathrm{h}, \mathrm{p}: \mathrm{X} \rightarrow \mathrm{Y}$ be a mapping such that $\mathrm{g}(0)=\mathrm{h}(0)=\mathrm{p}(0)=0$ and

$$
N(f(x+y)+f(x-y)+g(z)+h(l), t) \geqslant N\left(k p\left(\frac{2 x+z+l}{k}\right), t\right)
$$

for all $x, y, z \in X$. Then the mapping $f, g$ and $h$ are additive, for all $x \in X$. 
Proof. Letting $x=y=z=l=0$ in (2.1), we get

$$
N(2 f(0), t) \geqslant N(k p(0), t)=1,
$$

$f(0)=0$.

Letting $(x, y, z, l)=(x, x, 0,-2 x)$ in $(2.1)$, we get

$$
N(f(2 x)+h(-2 x), t) \geqslant N(K p(0), t)=1
$$

for all $x \in X$.

Replacing $(x, y, z, l)$ by $(x, x,-2 x, 0)$ in $(2.1)$, we get

$$
N(f(2 x)+g(-2 x), t) \geqslant N(K p(0), t)=1
$$

for all $x \in X$.

Replacing $(x, y, z, l)$ by $(x, y,-x,-x)$ in $(2.1)$, we get

$$
N(f(x+y)+f(x-y)+g(-x)+h(-x), t) \geqslant N(K p(0), t)=1
$$

for all $x, y \in X$.

By (2.2), (2.3) and (2.4), we get

$$
f(x+y)+f(x-y)-2 f(x)=0
$$

for all $x, y \in X$. Hence $f(x)$ is additive mapping for all $x, y \in X$. Since $f$ is additive, it is clear that $g$ and $h$ are additive; and $g(x)=-f(-x), h(x)=-f(-x)$, as desired.

Next, we show that the Hyers-Ulam stability of the functional inequality (1.3).

Theorem 2.2. Assume that a mapping $\mathrm{f}, \mathrm{g}, \mathrm{h}, \mathrm{p}: \mathrm{X} \rightarrow \mathrm{Y}$ with $\mathrm{g}(0)=\mathrm{h}(0)=\mathrm{p}(0)=0$ satisfies the inequality

$$
N\left(f(x+y)+f(x-y)+g(z)+h(l)-K p\left(\frac{2 x+z+l}{K}\right), t\right) \geqslant \frac{t}{t+\phi(x, y, z, l)},
$$

where $\phi: X^{4} \rightarrow[0, \infty)$ is a function such that

$$
\frac{1}{2} \phi(2 x, 2 y, 2 z, 2 l) \leqslant \phi(x, y, z, l)
$$

for all $x, y, z, l \in X$. Then there exists a unique additive mapping $A: X \rightarrow Y$ such that

$$
\begin{aligned}
N(f(x)-A(x), t) \geqslant \min & \left\{\frac{2 t}{2 t+3 \phi(x, x,-x,-x)}, \frac{2 t}{2 t+3 \phi(x / 2, x / 2,-x, 0)},\right. \\
N(g(x)-A(x), t) \geqslant \min & \left\{\frac{2 t}{2 t+3 \phi(x / 2, x / 2,0,-x)}\right\}, \\
N(h(x)-A(x), t) \geqslant \min & \left\{\frac{2 t}{2 t+3 \phi(x / 2, x / 2,0,-x)}\right\}, \\
& \left.\frac{2 t}{2 t+3 \phi(x, x,-x,-x)}, \frac{2 t}{2 t+3 \phi(x / 2, x / 2,0,-x)}\right\}
\end{aligned}
$$

for all $x \in X$. 
Proof. Letting $x=y=z=l=0$ in (2.6), we get $N(2 f(0)-K p(0), t) \geqslant \frac{t}{t+\phi(0,0,0,0)}=1$. So $f(0)=0$.

Letting $(x, y, z, l)=(x, y,-2 x, 0)$ in (2.6), we get

$$
N(f(2 x)+g(-2 x), t) \geqslant \frac{t}{t+\phi(x, x,-2 x, 0)}
$$

for all $x \in X$.

Replacing $(x, y, z, l)$ by $(x, y, 0,-2 x)$ in (2.6), we get

$$
N(f(2 x)+h(-2 x), t) \geqslant \frac{t}{t+\phi(x, x, 0,-2 x)}
$$

for all $x \in X$.

Replacing $(x, y, z, l)$ by $(x, x,-x,-x)$ in (2.6), we get

$$
N(f(x+y)+f(x-y)+g(-x)+h(-x), t) \geqslant \frac{t}{t+\phi(x,, y,-x,-x)}
$$

for all $x \in X$.

By (2.8), (2.9) and (2.10), it follows that

$$
\begin{gathered}
N(2 f(x)-f(2 x), t) \geqslant \min \left\{\frac{t}{t+3 \phi(x, x,-x,-x)}, \frac{t}{t+3 \phi\left(\frac{x}{2}, \frac{x}{2},-x, 0\right)},\right. \\
\left.\frac{t+3 \phi\left(\frac{x}{2}, \frac{x}{2}, 0,-x,\right)}{t+1}\right\}
\end{gathered}
$$

for all $x \in X$ such that

$$
\begin{gathered}
N\left(f(x)-\frac{1}{2} f(2 x), t\right) \geqslant \min \left\{\frac{2 t}{2 t+3 \phi(x, x,-x,-x)}, \frac{2 t}{2 t+3 \phi\left(\frac{x}{2}, \frac{x}{2},-x, 0\right)},\right. \\
\left.\frac{2 t}{2 t+3 \phi\left(\frac{x}{2}, \frac{x}{2}, 0,-x,\right)}\right\}
\end{gathered}
$$

for all $x \in X$.

It follows from (2.12) that

$$
\begin{aligned}
& N\left(\frac{1}{2^{n}} f\left(2^{n} x\right)-\frac{1}{2^{m}} f\left(2^{m} x\right),(m-n) t\right)=N\left(\sum_{j=k}^{m-1}\left[\frac{1}{2^{j}} f\left(2^{j} x\right)-\frac{1}{2^{j+1}} f\left(2^{j+1} x\right)\right],(m-n) t\right) \\
& \geqslant \min \bigcup_{j=k}^{m-1}\left\{N\left(\frac{1}{2^{j}} f\left(2^{j} x\right)-\frac{1}{2^{j+1}} f\left(2^{j+1} x\right), t\right)\right\} \\
& \geqslant \min \left\{\frac{2 t}{2 t+3 \frac{1}{2^{n}} \phi\left(2^{n} x, 2^{n} x,-2^{n} x,-2^{n} x\right)^{\prime}},\right. \frac{2 t}{2 t+3 \frac{1}{2^{n}} \phi\left(2^{n-1} x, 2^{n-1} x,-2^{n} x \cdot 0\right)^{\prime}}, \\
&\left.\frac{2 t}{2 t+3 \frac{1}{2^{n}} \phi\left(2^{n-1} x, 2^{n-1} x, 0,-2^{n} x\right)}\right\}
\end{aligned}
$$

for all nonnegative integers $m$ and $n$ with $m>n$ and all $x \in X$. It means that the sequence $\left\{\left(\frac{c}{a}\right)^{n} f\left(\left(\frac{a}{c}\right)^{n} x\right)\right\}$ is a Cauchy sequence for all $x \in X$. Since $Y$ is complete, the sequence $\left\{\left(\frac{1}{2}\right)^{n} f\left(2^{n} x\right)\right\}$ converges. We define 
the mapping $A: X \rightarrow Y$ by $A(x)=\lim _{n \rightarrow \infty}\left\{\left(\frac{1}{2}\right)^{n} f\left(2^{n} x\right)\right\}$ for all $x \in X$. Moreover, we get

$$
\begin{gathered}
N(f(x)-A(x), t) \geqslant \min \left\{\frac{2 t}{2 t+3 \phi(x, x,-x,-x)}, \frac{2 t}{2 t+3 \phi(x / 2, x / 2,-x, 0)},\right. \\
\left.\frac{2 t}{2 t+3 \phi(x / 2, x / 2,0,-x)}\right\}
\end{gathered}
$$

for all $x \in X$.

Similarly, there exists a mapping $B: X \rightarrow Y$ such that $B(x)=\lim _{n \rightarrow \infty} \frac{1}{2^{n}} g\left(2^{n} x\right)$ and

$$
\begin{gathered}
N(g(x)-B(x), t) \geqslant \min \left\{\frac{2 t}{2 t+3 \phi(x, x,-x,-x)}, \frac{2 t}{2 t+3 \phi(x / 2, x / 2,-x, 0)},\right. \\
\left.\frac{2 t}{2 t+3 \phi(x / 2, x / 2,0,-x)}\right\}
\end{gathered}
$$

for all $x \in X$.

We also obtain a mapping $C: X \rightarrow Y$ such that $C(x):=\lim _{n \rightarrow \infty} \frac{1}{2^{n}} h\left(2^{n} x\right)$, and

$$
\begin{gathered}
N(h(x)-C(x), t) \geqslant \min \left\{\frac{2 t}{2 t+3 \phi(x, x,-x,-x)}, \frac{2 t}{2 t+3 \phi(x / 2, x / 2,-x, 0)},\right. \\
\left.\frac{2 t}{2 t+3 \phi(x / 2, x / 2,0,-x)}\right\}
\end{gathered}
$$

for all $x \in X$.

Next, we show that $A$ is an Jensen mapping.

$$
\begin{aligned}
& N\left(\frac{1}{2^{n}} f\left(2^{n} x+2^{n} y\right)+\frac{1}{2^{n}} f\left(2^{n} x-2^{n} y\right)-2 \frac{1}{2^{n}} f\left(2^{n} x\right), 3 t\right) \\
&=N\left(\frac{1}{2^{n}} f\left(2^{n} x+2^{n} y\right)\right. \\
&+\frac{1}{2^{n}} f\left(2^{n} x-2^{n} y\right)+\frac{1}{2^{n}} g\left(-2^{n} x\right)+\frac{1}{2^{n}} h\left(-2^{n} x\right) \\
&\left.-\frac{1}{2^{n}} g\left(-2^{n} x\right)-\frac{1}{2^{n}} h\left(-2^{n} x\right)-2 \frac{1}{2^{n}} f\left(2^{n} x\right), 3 t\right) \\
& \geqslant \min \left\{1, \frac{t}{t+\frac{1}{2^{n}} \phi\left(2^{n-1} x, 2^{n-1} x, 0,-2^{n} x\right)},\right. \\
&\left.\frac{t}{t+\frac{1}{2^{n}} \phi\left(2^{n-1} x, 2^{n-1} x,-2^{n} x, 0\right)}\right\}
\end{aligned}
$$

for all $x, y \in X$. Thus the mapping $A: X \rightarrow Y$ is additive.

Now, we prove the uniqueness of $A$. Assume that $T: X \rightarrow Y$ is another additive mapping satisfying (2.7). We obtain

$$
\begin{gathered}
N(A(x)-T(x), t)=N\left(\frac{1}{2^{n}} A\left(2^{n} x\right)-\frac{1}{2^{n}} T\left(2^{n} x\right), t\right) \\
\geqslant \min \left\{\frac{2 t}{2 t+\frac{3}{2^{n}} \phi\left(2^{n} x, 2^{n} x,-2^{n} x,-2^{n} x\right)}, \frac{2 t}{2 t+\frac{3}{2^{n}} \phi\left(2^{n-1} x, 2^{n-1} x,-2^{n} x, 0\right)},\right. \\
\left.\frac{2 t}{2 t+\frac{3}{2^{n}} \phi\left(2^{n-1} x, 2^{n-1} x, 0,-2^{n} x\right)}\right\},
\end{gathered}
$$

which tends to zero as $n \rightarrow \infty$ for all $x \in X$. Then we can conclude that $A(x)=T(x)$ for all $x \in X$.

Therefore the inequalities (2.7) hold. 
Corollary 2.3. Let $\mathrm{q}$ and $\theta$ be positive real numbers with $0<\mathrm{q}<1$. Let $\mathrm{f}, \mathrm{g}, \mathrm{h}, \mathrm{l}: \mathrm{X} \rightarrow \mathrm{Y}$ be the mappings with $\mathrm{g}(0)=\mathrm{h}(0)=\mathrm{p}(0)=0$ satisfying

$$
N\left(f(x+y)+f(x-y)+g(z)+h(l)-k p\left(\frac{2 x+y+z+l}{k}\right), t\right) \geqslant \frac{t}{t+\theta\left(\|x\|^{p}+\|y\|^{p}+\|z\|^{p}+\|l\|^{p}\right)}
$$

for all $x, y, z \in X$. Then there exists a unique additive mapping $A: X \rightarrow Y$ such that

$$
\begin{aligned}
& N(f(x)-A(x), t) \geqslant \frac{t}{t+3 \theta\|x\|^{p}}, \\
& N(g(x)-A(x), t) \geqslant \frac{t}{t+3 \theta\|x\|^{p}}, \\
& N(h(x)-A(x), t) \geqslant \frac{t}{t+3 \theta\|x\|^{p}}
\end{aligned}
$$

for all $x \in X$.

\section{Hyers-Ulam stability of the functional inequality (1.4)}

Throughout this section, assume that $k$ is nonzero real numbers.

Proposition 3.1. Let $\mathrm{f}, \mathrm{g}, \mathrm{h}, \mathrm{p}: \mathrm{X} \rightarrow \mathrm{Y}$ be a mapping with $\mathrm{g}(0)=\mathrm{h}(0)=\mathrm{p}(0)=0$ such that

$$
N(f(x+y)+f(x-y)+g(z)+k h(l), t) \geqslant N\left(k p\left(\frac{2 x+z}{k}+l\right), t\right)
$$

for all $x, y, z \in X$. Then the mapping $f: X \rightarrow Y$ is additive.

Proof. Letting $x=y=z=0$ in (3.1), we get

$$
N(2 f(0), t) \geqslant N(p(0), t)=1
$$

So $f(0)=0$.

Letting $(x, y, z, l)=(x, x,-2 x, 0)$ in (3.1), we get

$$
N(f(2 x)+g(-2 x), t) \geqslant N(k p(0), t)=1
$$

for all $x \in X$. So $f(x)=-g(-x)$ for all $x \in X$.

Replacing $(x, y, z, l)$ by $\left(x, x, 0,-\frac{2 x}{k}\right)$ in (3.1), we get

$$
N\left(f(2 x)+k h\left(\frac{2}{k} x\right), t\right) \geqslant N(k p(0), t)=1
$$

for all $x \in X$. So $f(x)=-k h\left(-\frac{x}{k}\right)$ for all $x \in X$.

Thus we get

$$
\begin{aligned}
N(f(x+y)+f(x-y)-2 f(x), 3 t)= & N(f(x+y)+f(x-y)+g(x)+k h(x) \\
& -g(x)-k h(x)-2 f(x), 3 t) \\
\geqslant & \min \{N(k p(0), t), N(f(x)+g(x), t), N(f(x)+k h(x / k), t)\}
\end{aligned}
$$

for all $x, y \in X$. So $f(x+y)+f(x-y)-2 f(x)=0$ for all $x, y \in X$. Similarly, we can show that

$$
g(x+y)+g(x-y)-2 g(x)=0,
$$

and $h(x+y)+h(x-y)-2 h(x)=0$, as desired. 
Theorem 3.2. Assume that the mappings $\mathrm{f}, \mathrm{g}, \mathrm{h}, \mathrm{p}: \mathrm{X} \rightarrow \mathrm{Y}$ with $\mathrm{g}(0)=\mathrm{h}(0)=\mathrm{p}(0)=0$ satisfying the inequality

$$
N\left(f(x+y)+f(x-y)+g(z)+k h(l)-k p\left(\frac{2 x+z}{k}+l\right), t\right) \geqslant \frac{t}{t+\phi(x, y, z, l)},
$$

where $\phi: X^{4} \rightarrow[0, \infty)$ satisfies $\phi(0,0,0,0)=0$ and

$$
\widetilde{\phi}(x, y, z, l):=\sum_{j=1}^{\infty} \frac{1}{2^{j}} \phi\left(2^{j} x, 2^{j} y, 2^{j} z\right)<\infty
$$

for all $\mathrm{x}, \mathrm{y}, \mathrm{z}, \mathrm{l} \in \mathrm{X}$. Then there exists a unique additive mapping $\mathrm{A}: \mathrm{X} \rightarrow \mathrm{Y}$ such that

$$
\begin{aligned}
& N(f(x)-A(x), t) \geqslant \min \left\{\frac{2 t}{2 t+3 \phi\left(x, x, 0,-\frac{2 x}{k}\right)}, \frac{2 t}{2 t+3 \phi(x, x,-2 x, 0)}, \frac{2 t}{2 t+3 \phi\left(x, x,-x,-\frac{x}{k}\right)}\right\}, \\
& N(g(x)+A(-x), t) \geqslant \min \left\{\frac{2 t}{2 t+3 \phi\left(-x,-x, 0, \frac{2 x}{k}\right)}, \frac{2 t}{2 t+3 \phi(-x,-x, 2 x, 0)},\right. \\
& N\left(h(x)+\frac{1}{k} A(-k x), t\right) \geqslant \min \left\{\frac{2 t}{2 t+3 \phi\left(-x,-x, x, \frac{x}{k}\right)}\right\}, \\
& \\
& \frac{2 t+3 \phi(-k x,-k x, 0,2 x)}{2 t+3 \phi(-k x,-k x, k x, x)}, \frac{2 t}{2 t+3 \phi(-k x,-k x, 2 k x, 0)},
\end{aligned}
$$

for all $x \in X$.

Proof. Letting $x=y=z=l=0$ in (3.4), we get $N(2 f(0), t) \geqslant \frac{t}{t+\phi(0,0,0,0)}$. So $f(0)=0$.

Letting $y=x, z=0, l=-\frac{2 x}{k}$ in (3.4), we obtain

$$
N\left(f(2 x)+k h\left(-\frac{2 x}{k}\right), t\right) \geqslant \frac{t}{t+\phi\left(x, x, 0,-\frac{2 x}{k}\right)}
$$

for all $x \in X$.

Letting $y=x, z=-2 x, l=0$ in (3.4), we obtain

$$
N(f(2 x)+g(-2 x), t) \geqslant \frac{t}{t+\phi(x, x,-2 x, 0)}
$$

for all $x \in X$.

Letting $y=x, z=-x, l=-\frac{x}{k}$ in (3.4), we get

$$
N\left(f(2 x)+g(-x)+k h\left(-\frac{x}{k}\right), t\right) \geqslant \frac{t}{t+\phi\left(x, x,-x, \frac{x}{k}\right)}
$$

for all $x \in X$. So

$$
\begin{aligned}
N(f(2 x)- & 2 f(x), 3 t) \\
& =N\left(f(2 x)+g(-x)+k h\left(-\frac{x}{k}\right)-g(-x)-k h\left(-\frac{x}{k}\right)-2 f(x), 3 t\right) \\
& \geqslant \min \left\{N\left(f(2 x)+g(-x)+k h\left(-\frac{x}{k}\right), t\right), N(f(x)+g(-x), t), N\left(f(x)+k h\left(-\frac{x}{k}\right), t\right\}\right. \\
& \geqslant \min \left\{\frac{t}{t+\phi\left(x, x, 0,-\frac{2 x}{k}\right)}, \frac{t}{t+\phi(x, x,-2 x, 0)}, \frac{t}{t+\phi\left(x, x,-x,-\frac{x}{k}\right)}\right\}
\end{aligned}
$$


for all $x \in X$. Thus,

$$
N\left(f(x)-\frac{1}{2} f(2 x), t\right) \geqslant \min \left\{\frac{2 t}{2 t+3 \phi\left(x, x, 0,-\frac{2 x}{k}\right)}, \frac{2 t}{2 t+3 \phi(x, x,-2 x, 0)}, \frac{2 t}{2 t+3 \phi\left(x, x,-x,-\frac{x}{k}\right)}\right\} .
$$

It follows from (3.9) that

$$
\begin{aligned}
& N\left(\frac{1}{2^{n}} f\left(2^{n} x\right)-\frac{1}{2^{m}} f\left(2^{m} x\right),(m-n) t\right)=N\left(\sum_{j=k}^{m-1}\left[\frac{1}{2^{j}} f\left(2^{j} x\right)-\frac{1}{2^{j+1}} f\left(2^{j+1} x\right)\right],(m-n) t\right) \\
& \geqslant \min \bigcup_{j=k}^{m-1}\left\{N\left(\frac{1}{2^{j}} f\left(2^{j} x\right)-\frac{1}{2^{j+1}} f\left(2^{j+1} x\right), t\right)\right\} \\
& \frac{2 t}{2 t+3 \frac{1}{2^{n}} \phi\left(2^{n-1} x, 2^{n-1} x, 0,-\frac{2^{n} x}{k}\right)^{\prime}}, \frac{2 t}{2 t+\frac{1}{2^{n}} \phi\left(2^{n-1} x, 2^{n-1} x,-2^{n} x \cdot 0\right)^{\prime}}, \\
&\left.\frac{2 t+3 \frac{1}{2^{n}} \phi\left(2^{n-1} x, 2^{n-1} x,-2^{n-1} x,-\frac{2^{n-1} x}{k}\right)}{2 t}\right\}
\end{aligned}
$$

for all nonnegative integers $m$ and $n$ with $m>n$ and all $x \in X$. It means that the sequence $\left\{\frac{1}{2^{n}} f\left(2^{n} x\right)\right\}$ is a Cauchy sequence for all $x \in X$. Since $Y$ is complete, the sequence $\left.\left\{\frac{1}{2^{n}}\right) f\left(2^{n} x\right)\right\}$ converges. So we may define the mapping $A: X \rightarrow Y$ by $A(x)=\lim _{n \rightarrow \infty} \frac{1}{2^{n}} f\left(2^{n} x\right)$ for all $x \in X$.

Moreover, we get the first formula of (3.5). By the (3.6) and (3.7), we get the second and third formula of (3.5).

Next, we show that $A$ is an Jensen mapping.

$$
\begin{aligned}
N\left(\frac{1}{2^{n}} f\left(2^{n} x+2^{n} y\right)+\right. & \left.\frac{1}{2^{n}} f\left(2^{n} x-2^{n} y\right)-2 \frac{1}{2^{n}} f\left(2^{n} x\right), 3 t\right) \\
= & N\left(\frac{1}{2^{n}} f\left(2^{n} x+2^{n} y\right)+\frac{1}{2^{n}} f\left(2^{n} x-2^{n} y\right)+\frac{1}{2^{n}} g\left(-2^{n} x\right)+\frac{k}{2^{n}} h\left(-2^{n} x\right)\right. \\
& \left.-\frac{1}{2^{n}} g\left(-2^{n} x\right)-\frac{k}{2^{n}} h\left(-2^{n} x\right)-2 \frac{1}{2^{n}} f\left(2^{n} x\right), 3 t\right) \\
\geqslant & \min \left\{1, \frac{t}{t+\frac{1}{2^{n}} \phi\left(2^{n+1} x, 2^{n+1} x, 0,-\frac{2^{n} x}{k}\right)}, \frac{t}{t+\frac{1}{2^{n}} \phi\left(2^{n+1} x, 2^{n+1} x,-2^{n} x, 0\right)}\right\}
\end{aligned}
$$

for all $x, y \in X$. Thus the mapping $A: X \rightarrow Y$ is additive. So the mapping $A: X \rightarrow Y$ is an additive mapping.

The rest of the proof of theorem similar with Theorem 2.2.

Corollary 3.3. Let $\mathrm{q}, \theta$ and $\mathrm{k}$ be positive real numbers with $\mathrm{q}>1$. Let $\mathrm{f}, \mathrm{h}, \mathrm{g}, \mathrm{l}: \mathrm{X} \rightarrow \mathrm{Y}$ be the mappings with $\mathrm{h}(0)=\mathrm{g}(0)=\mathrm{p}(0)$ satisfying

$$
\left.\mathrm{N}\left(\mathrm{f}(x+y)+f(x-y)+g(z)+k h(l)-k p\left(\frac{2 x+z}{k}+l\right), t\right) \geqslant \frac{t}{t+\theta\left(\|x\|^{q}+\|y\|^{q}+\|z\|^{q}+\|l\| q\right.}\right)
$$

for all $x, y, z \in X$. Then there exists a unique additive mapping $A: X \rightarrow Y$ such that

$$
N(f(x)-A(x), t) \geqslant \min \left\{\frac{2 t}{2 t+3 \theta\|x\|^{q}\left(3+\frac{1}{k^{q}}\right)}, \frac{2 t}{2 t+3 \theta\|x\|^{q}\left(2+\frac{2^{q}}{k^{q}}\right)}, \frac{2 t}{2 t+3 \theta\|x\|^{q}\left(2+2^{q}\right)}\right\},
$$




$$
\begin{aligned}
& N(g(x)+A(-x), t) \geqslant \min \left\{\frac{2 t}{2 t+3 \theta\|x\|^{q}\left(3+\frac{1}{k^{q}}\right)}, \frac{2 t}{2 t+3 \theta\|x\|^{q}\left(2+\frac{2 q}{k^{q}}\right)}, \frac{2 t}{2 t+3 \theta\|x\|^{q}\left(2+2^{q}\right)}\right\} \\
& N\left(h(x)+\frac{1}{k} A(-k x)\right) \geqslant \min \left\{\frac{2 t}{2 t+3 \theta\|x\|^{q}\left(3+\frac{1}{k^{q}}\right)}, \frac{2 t}{2 t+3 \theta\|x\|^{q}\left(2+\frac{2^{q}}{k^{q}}\right)}, \frac{2 t}{2 t+3 \theta\|x\|^{q}(2+2 q)}\right\}
\end{aligned}
$$

for all $x \in X$.

\section{Acknowledgment}

This work was supported by National Natural Science Foundation of China (No. 11761074), the projection of the Department of Science and Technology of JiLin Province and the Education Department of Jilin Province (No. 20170101052JC) and Natural Science Fund of Liaoning Province (No. 201602547).

\section{References}

[1] H. Azadi-Kenary, J. R. Lee, C. Park, Non-Archimedean stability of an AQQ-functional equation, J. Comput. Anal. Appl., 14 (2012), 211-227. 1

[2] T. Bag S. K. Samanta, Finite dimensional fuzzy normed linear spaces, J. Fuzzy Math., 11 (2003), 687-705. 1

[3] V. Balopoulos, A. G. Hatzimichailidis, B. K. Papadopoulos, Distance and similarity measures for fuzzy operators, Inform. Sci., 177 (2007), 2336-2348. 1

[4] I.-S. Chang, Stability of higher ring derivations in fuzzy Banach algebras, J. Computat. Anal. Appl., 14 (2012), 10591066. 1

[5] S. C. Cheng, J. N. Mordeson, Fuzzy linear operator and fuzzy normed linear spaces, Bull. Calcutta Math. Soc., 86 (1994), 429-436. 1

[6] I. G. Cho, D. S. Kang, H. J. Koh, Stability problems of cubic mappings with the fixed point alternative, J. Computat. Anal. Appl., 14 (2012), 132-142. 1

[7] Y. J. Cho, C. Park, R. Saadati, Functional inequalities in non-Archimedean Banach spaces, Appl. Math. Lett., 23 (2010), 1238-1242. 1

[8] M. Eshaghi Gordji, M. Bavand Savadkouhi, M. Bidkham, Stability of a mixed type additive and quadratic functional equation in non-Archimedean spaces, J. Comput. Anal. Appl., 12 (2010), 454-462. 1

[9] M. Eshaghi Gordji, A. Bodaghi, On the stability of quadratic double centralizers on Banach algebras, J. Computat. Anal. Appl., 13 (2011), 724-729.

[10] M. Eshaghi Gordji, R. Farokhzad Rostami, S. A. R. Hosseinioun, Nearly higher derivations in unital C*-algebras, J. Computat. Anal. Appl., 13 (2011), 734-742.

[11] M. Eshaghi Gordji, S. Kaboli Gharetapeh, T. Karimi, E. Rashidi, M. Aghaei, Ternary Jordan derivations on C*-ternary algebras, J. Computat. Anal. Appl., 12 (2010), 463-470. 1

[12] M. Fang, G. Lu, D. H. Pei, Functional inequalities in generalized quasi-Banach spaces, J. Nonlinear Sci. Appl., 9 (2016), 2481-2491. 1

[13] C. Felbin, Finite dimensional fuzzy normed linear space, Fuzzy Sets and Systems, 48 (1992), 239-248. 1

[14] Z. Gajda, On stability of additive mappings, Internat. J. Math. Math. Sci., 14 (1991), 431-434. 1

[15] P. Gǎvruta, A generalization of the Hyers-Ulam-Rassias stability of approximately additive mappings, J. Math. Anal. Appl., 184 (1994), 431-436. 1

[16] D. H. Hyers, On the stability of the linear functional equation, Proc. Natl. Acad. Sci. U. S. A., 27 (1941), 222-224. 1

[17] S.-M. Jung, Hyers-Ulam-Rassias Stability of Functional Equations in Mathematical Analysis, Hadronic Press Inc., Palm Harbor, (2001). 1

[18] A. K. Katsaras, Fuzzy topological vector spaces II, Fuzzy Sets and Systems, 12 (1984), 143-154. 1

[19] S. V. Krishna, K. K. M. Sarma, Separation of fuzzy normed linear spaces, Fuzzy Sets and Systems, 63 (1994), $207-217$. 1

[20] G. Lu, Y. Jiang, C. Park, Functional inequality in Fréchet spaces, J. Comput. Anal. Appl., 15 (2013), 369-373. 1

[21] G. Lu, C. Park, Additive functional inequalities in Banach spaces, J. Inequal. Appl., 2012 (2012), 10 pages. 1

[22] G. Lu, C. Park, Hyers-Ulam stability of additive set-valued functional equations, Appl. Math. Lett., 24 (2011), $1312-1316$. 1

[23] P. Malliavin, Stochastic Analysis, Springer-Verlag, Berlin, (1997). 1

[24] C.-G. Park, Homomorphisms between Poisson JC*-algebra, Bull. Braz. Math. Soc. (N.S.), 36 (2005), 79-97. 1

[25] C. Park, Hyers-Ulam-Rassias stability of homomorphisms in quasi-Banach algebras, Bull. Sci. Math., 132 (2008), 87-96.

[26] C. Park, Y. J. Cho, H. Azadi-Kenary, Orthogonal stability of a generalized quadratic functional equation in nonArchimedean spaces, J. Computat. Anal. Appl., 14 (2012), 526-535. 1 
[27] C. Park, Y. S. Cho, M.-H. Han, Functional inequalities associated with Jordan-von Neumann type additive functional equations, J. Inequal. Appl., 2007 (2007), 13 pages. 1

[28] C. Park, S. Y. Jang, R. Saadati, Fuzzy approximate of homomorphisms, J. Comput. Anal. Appl., 14 (2012), 833-841. 1

[29] T. M. Rassias, On the stability of the linear mapping in Banach spaces, Proc. Amer. Math. Soc., 72 (1978), 297-300. 1

[30] J. M. Rassias, On approximation of approximately linear mappings by linear mappings, Bull. Sci. Math. (2), 108 (1984), 445-446. 1, 1.2

[31] S. Shagholi, M. Eshaghi Gordji, M. Bavand Savadkouhi, Nearly ternary cubic homomorphism in ternary Fréchet algebras, J. Comput. Anal. Appl., 13 (2011), 1106-1114. 1

[32] S. Shagholi, M. Eshaghi Gordji, M. Bavand Savadkouhi, Stability of ternary quadratic derivations on ternary Banach algebras, J. Comput. Anal. Appl., 13 (2011), 1097-1105. 1

[33] S. M. Ulam, A Collection of the Mathematical Problems, Interscience Publ., New York, (1960). 1

[34] J.-Z. Xiao, X.-H. Zhu, Fuzzy normed spaces of operators and its completeness, Fuzzy Sets and Systems, 133 (2003), 389-399. 1 